PROCEEDINGS OF THE

AMERICAN MATHEMATICAL SOCIETY

Volume 130, Number 12, Pages 3597-3604

S 0002-9939(02)06628-5

Article electronically published on May 8, 2002

\title{
TRIANGULAR TOEPLITZ CONTRACTIONS AND COWEN SETS FOR ANALYTIC POLYNOMIALS
}

\author{
MUNEO CHŌ, RAÚL E. CURTO, AND WOO YOUNG LEE \\ (Communicated by David R. Larson)
}

\begin{abstract}
Let $\mathfrak{L}_{N}$ be the collection of $N \times N$ lower triangular Toeplitz matrices and let $\mathfrak{T}_{N}$ be the collection of $N \times N$ lower triangular Toeplitz contractions. We show that $\mathfrak{T}_{N}$ is compact and strictly convex, in the spectral norm, with respect to $\mathfrak{L}_{N}$; that is, $\mathfrak{T}_{N}$ is compact, convex and $\partial_{\mathfrak{L}_{N}} \mathfrak{T}_{N} \subseteq \operatorname{ext} \mathfrak{T}_{N}$, where $\partial_{\mathfrak{L}_{N}}(\cdot)$ and $\operatorname{ext}(\cdot)$ denote the topological boundary with respect to $\mathfrak{L}_{N}$ and the set of extreme points, respectively. As an application, we show that the reduced Cowen set for an analytic polynomial is strictly convex; more precisely, if $f$ is an analytic polynomial and if $G_{f}^{\prime}:=\left\{g \in H^{\infty}(\mathbb{T})\right.$ : $g(0)=0$ and the Toeplitz operator $T_{f+\bar{g}}$ is hyponormal $\}$, then $G_{f}^{\prime}$ is strictly convex. This answers a question of $\mathrm{C}$. Cowen for the case of analytic polynomials.
\end{abstract}

\section{Introduction}

Let $M_{n}(\mathbb{C})$ denote the set of $n \times n$ complex matrices. If $A \in M_{n}(\mathbb{C})$, then the singular values of $A$ are defined by the nonnegative square roots of the eigenvalues of $A^{*} A$. The spectral norm of $A,\|A\|$, is defined as the largest singular value of $A$, i.e., $\|A\|=\max \left\{\sqrt{\lambda}: \lambda \in \sigma\left(A^{*} A\right)\right\}$. It is well known that the spectral norm coincides with the operator norm, that is, $\|A\|=\sup _{\|x\|=1}\|A x\|$. Thus, $\|A\| \leq$ $1 \Longleftrightarrow \sigma\left(A^{*} A\right) \subseteq[0,1]$, and $\|A\|=1 \Longleftrightarrow 1 \in \sigma\left(A^{*} A\right)$. Let $\mathfrak{L}_{N}$ be the collection of $N \times N$ lower triangular Toeplitz matrices and let $\mathfrak{T}_{N}$ be the collection of $N \times N$ lower triangular contractive Toeplitz matrices (or simply Toeplitz contractions). An element $A$ of $\mathfrak{T}_{N}$ is of the form

$$
A=\left(\begin{array}{ccccc}
c_{0} & 0 & 0 & \ldots & 0 \\
c_{1} & c_{0} & \ddots & & \vdots \\
\vdots & \ddots & \ddots & \ddots & \vdots \\
c_{N-2} & & \ddots & \ddots & 0 \\
c_{N-1} & c_{N-2} & \ldots & c_{1} & c_{0}
\end{array}\right)
$$

Received by the editors September 7, 2000 and, in revised form, July 2, 2001.

2000 Mathematics Subject Classification. Primary 47B35, 15A57, 15A60; Secondary 47B20, 30D50.

Key words and phrases. Triangular Toeplitz contractions, hyponormal Toeplitz operators.

The second author's work was partially supported by NSF research grant DMS-9800931.

The third author's work was partially supported by KOSEF research project No. R01-200000003. 
with $\|A\| \leq 1$. In this article we establish that $\mathfrak{T}_{N}$ is strictly convex with respect to $\mathfrak{L}_{N}$; that is, the topological boundary of $\mathfrak{T}_{N}$ with respect to $\mathfrak{L}_{N}, \partial_{\mathfrak{L}_{N}} \mathfrak{T}_{N}$, consists entirely of extreme points (Theorem 1). Our proof relies on the CarathéodorySchur interpolation problem. As a consequence, we show that the reduced Cowen set of an analytic polynomial is strictly convex (Theorem 9). This is related to the contractive completion problem for partially given triangular Toeplitz matrices, which has been considered by C.R. Johnson and L. Rodman [JR] and G. Nævdal [Næ.

\section{LOWER triangular TOePlitz CONTRACTIONS}

Consider first the following interpolation problem, called the Carathéodory-Schur interpolation problem (CSIP). Given $c_{0}, c_{1}, \cdots, c_{N-1}$ in $\mathbb{C}$, find an analytic function $k$ on the open unit disc $\mathbb{D}$ such that

(i) $\widehat{k}(j)=c_{j}, \quad j=0, \cdots, N-1 \quad(\widehat{k}(j)$ denotes the $j$ th Fourier coefficient of $k)$, (ii) $\sup _{z \in \mathbb{D}}|k(z)| \leq 1$.

CSIP can be analyzed by a matricial argument (cf. [Sch]): CSIP is solvable if and only if the Toeplitz matrix

$$
C:=\left(\begin{array}{ccccc}
c_{0} & 0 & 0 & \ldots & 0 \\
c_{1} & c_{0} & \ddots & & \vdots \\
\vdots & \ddots & \ddots & \ddots & \vdots \\
c_{N-2} & & \ddots & \ddots & 0 \\
c_{N-1} & c_{N-2} & \ldots & c_{1} & c_{0}
\end{array}\right)
$$

is a contraction, that is, $\|C\| \leq 1$, or equivalently, $I-C C^{*} \geq 0$. Today this result is also called the Carathéodory-Fejér Theorem. A proof of this result can be obtained by means of the Commutant Lifting Theorem (cf. GGK, Proposition XXVII.7.2]). In particular, by Pick's Theorem (cf. [Ga, Corollary 2.3]) CSIP has a unique solution $k$ if and only if $\operatorname{det}\left(I-C C^{*}\right)=0$. Moreover, in the cases where $\operatorname{det}\left(I-C C^{*}\right)=0$, the unique solution is a finite Blaschke product $k$ of the form

$$
k(z)=e^{i \theta} \prod_{j=1}^{n} \frac{z-\zeta_{j}}{1-\overline{\zeta_{j}} z} \quad\left(\left|\zeta_{j}\right|<1 \text { for } j=1, \cdots, n ; \theta \in[0,2 \pi)\right),
$$

such that $\operatorname{deg}(k)=\operatorname{rank}\left(I-C C^{*}\right)$, where $\operatorname{deg}(k)$ denotes the degree of $k$, that is, the number of zeros of $k$ in the open unit disc $\mathbb{D}$ (see also [Ta, Theorem]).

We now have:

Theorem 1. Recall that $\mathfrak{L}_{N}$ is the collection of $N \times N$ lower triangular Toeplitz matrices and $\mathfrak{T}_{N}$ is the collection of $N \times N$ lower triangular Toeplitz contractions. Then $\mathfrak{T}_{N}$ is compact and strictly convex, in the spectral norm, with respect to $\mathfrak{L}_{N}$; that is, $\mathfrak{T}_{N}$ is compact, convex and

$$
\partial_{\mathfrak{L}_{N}} \mathfrak{T}_{N} \subseteq \operatorname{ext} \mathfrak{T}_{N}
$$

Proof. It is easy to see that $\mathfrak{T}_{N}$ is compact and convex. Moreover,

$$
\partial_{\mathfrak{L}_{N}} \mathfrak{T}_{N}=\left\{C \in \mathfrak{T}_{N}:\|C\|=1\right\}
$$


Towards strict convexity, recall that $\mathcal{K}$ is a strictly convex subset of a vector space $\mathcal{X}$ if

$$
x_{1}, x_{2} \in \mathcal{X} \text { and } \frac{1}{2}\left(x_{1}+x_{2}\right) \in \partial_{\mathcal{X}} \mathcal{K} \Longrightarrow x_{1} \notin \partial \mathcal{X} \mathcal{K}, \text { or } x_{2} \notin \partial \mathcal{X} \mathcal{K} \text {, or } x_{1}=x_{2}
$$

Equivalently, $\mathcal{K}$ is strictly convex if and only if $\partial_{\mathcal{X}} \mathcal{K} \subseteq$ ext $\mathcal{K}$. Suppose now that $C, D, \frac{1}{2}(C+D) \in \partial_{\mathfrak{L}_{N}} \mathfrak{T}_{N}$. It will suffice to show that $C=D$. Write

$$
C:=\left(\begin{array}{ccccc}
c_{0} & 0 & 0 & \ldots & 0 \\
c_{1} & c_{0} & \ddots & & \vdots \\
\vdots & \ddots & \ddots & \ddots & \vdots \\
c_{N-2} & & \ddots & \ddots & 0 \\
c_{N-1} & c_{N-2} & \ldots & c_{1} & c_{0}
\end{array}\right) \quad \text { and } \quad D:=\left(\begin{array}{ccccc}
d_{0} & 0 & 0 & \ldots & 0 \\
d_{1} & d_{0} & \ddots & & \vdots \\
\vdots & \ddots & \ddots & \ddots & \vdots \\
d_{N-2} & & \ddots & \ddots & 0 \\
d_{N-1} & d_{N-2} & \ldots & d_{1} & d_{0}
\end{array}\right) \text {. }
$$

Then by CSIP and the fact that $\|C\|=\|D\|=1$, there exist analytic functions $B_{i}$ $(i=1,2,3)$ on $\mathbb{D}$ such that

$$
\left\{\begin{array}{l}
\widehat{B_{1}}(j)=c_{j}, \quad \widehat{B_{2}}(j)=d_{j}, \quad \widehat{B_{3}}(j)=\frac{1}{2}\left(c_{j}+d_{j}\right) \quad(j=0, \cdots, N-1), \\
\sup _{z \in \mathbb{D}}\left|B_{i}(z)\right| \leq 1 \quad(i=1,2,3) .
\end{array}\right.
$$

Now recall that if $A \in M_{n}$ with $\|A\|=1$, then $\operatorname{det}\left(I-A A^{*}\right)=0$ : indeed if $\|A\|=1$, then $\left\|A A^{*}\right\|=1$, so $1 \in \sigma\left(A A^{*}\right)$, forcing $\operatorname{det}\left(I-A A^{*}\right)=0$. Thus by the preceding considerations and the assumption $\|C\|=\|D\|=\left\|\frac{1}{2}(C+D)\right\|=1$, we can see that each $B_{i}$ is a finite Blaschke product, that is, each is the unique solution of the associated interpolation problem (1). But since $\sup _{z \in \mathbb{D}}\left|\frac{1}{2}\left(B_{1}+B_{2}\right)(z)\right| \leq 1$ and $\frac{1}{2}\left(\widehat{B_{1}+B_{2}}\right)(j)=\frac{1}{2}\left(c_{j}+d_{j}\right)(j=0, \cdots, N-1)$, it follows from uniqueness that $\frac{1}{2}\left(B_{1}+B_{2}\right)=B_{3}$, which implies that $\frac{1}{2}\left(B_{1}+B_{2}\right)$ is a finite Blaschke product. Recall now that Blaschke products are extreme points of the unit ball of $H^{\infty}(\mathbb{T})$. In fact, by an argument of $\mathrm{K}$. deLeeuw and $\mathrm{W}$. Rudin [LR], if $f \in H^{\infty}$ and $\|f\|=1$, then $f$ is an extreme point of the unit ball of $H^{\infty}(\mathbb{T})$ if and only if $\int \log \left(1-\left|f\left(e^{i \theta}\right)\right|\right) d \theta=-\infty$, a condition Blaschke products satisfy. We thus conclude that $B_{1}=B_{2}$. So $c_{j}=d_{j}$ for $j=0, \cdots, N-1$, which implies $C=D$. This completes the proof.

Although our proof of Theorem 1 relies on the the Carathéodory-Fejér Theorem, we expect that a (finite) matricial argument can be found.

Corollary 2. For $S \in M_{n}(\mathbb{C})$, let $d_{S}:=\operatorname{dist}\left(S, \mathfrak{T}_{N}\right)$. Then

$$
m_{S}:=\left\{B \in \mathfrak{T}_{N}:\|S-B\|=d_{S}\right\}
$$

is compact, convex and nonempty.

Proof. Since $m_{0}=\{0\}$, we can assume $S \neq 0$. It is clear that $m_{S}$ is closed and nonempty (by Theorem 1), so we shall focus on convexity. Let $B, C \in m_{S}$, and let $0<t<1$. Since $t B+(1-t) C \in \mathfrak{T}_{N}$, we have

$$
\begin{aligned}
d_{S} & \leq\|t B+(1-t) C-S\|=\|t(B-S)+(1-t)(C-S)\| \\
& \leq t\|B-S\|+(1-t)\|C-S\|=d_{S} .
\end{aligned}
$$

It follows that $t B+(1-t) C \in m_{S}$, so $m_{S}$ is convex.

By a partial Toeplitz matrix we mean a Toeplitz matrix, some of whose entries are specified complex numbers, and whose remaining entries are unspecified. As a matter of fact, what one specifies or leaves unspecified are entire diagonals. 
Corollary 3. Let $A$ be a partial Toeplitz matrix whose strict upper triangular part is known and let $t c_{A}$ denote the set of Toeplitz completions $\widetilde{A}$ of $A$ such that (i) $\widetilde{A}-A$ is a Toeplitz contraction, and (ii) $\|\widetilde{A}\|$ has the smallest norm amongst Toeplitz completions. Then $t c_{A}$ is either a singleton or an infinite set.

Proof. Apply Corollary 2 with $A$ in place of $S$.

Write $\mathfrak{N}$ for the collection of $N \times N$ upper triangular matrices. Note that $\mathfrak{N}$ forms a nest algebra with the nest $\{0\} \subseteq\left\{e_{0}\right\} \subseteq \cdots \subseteq\left\{e_{0} \cdots, e_{N-1}\right\}$. Arveson's Distance Formula (cf. [Da, Theorem 9.5]) says that if $A=\left(c_{i-j}\right) \in \mathfrak{T}_{N}$, then

$$
\operatorname{dist}(A, \mathfrak{N})=\sup _{0 \leq l \leq N-2}\left\|P_{l}^{\perp} A P_{l}\right\|=\max _{1 \leq l \leq N-1}\left\|\left(c_{l+i-j}\right)_{1 \leq i \leq N-l, 1 \leq j \leq l}\right\|=: \alpha_{c},
$$

where $P_{l}$ is the projection onto $\left\{e_{0}, \cdots, e_{l}\right\}$. But if $\mathfrak{N}$ is replaced by $\mathfrak{T}_{N}^{\prime}$, the collection of $N \times N$ upper triangular Toeplitz contractions, then $\operatorname{dist}\left(A, \mathfrak{T}_{N}^{\prime}\right) \geq \alpha_{c}$. Thus, we need not expect that $\operatorname{dist}\left(A, \mathfrak{T}_{N}^{\prime}\right)=\alpha_{c}$ if $N>2$. However, if $A \in \mathfrak{T}_{N}$ satisfies dist $\left(A, \mathfrak{T}_{N}^{\prime}\right)=\alpha_{c}$, then by Corollary 3 , the solution $S \in \mathfrak{T}_{N}^{\prime}$ of the equation $\operatorname{dist}\left(A, \mathfrak{T}_{N}^{\prime}\right)=\|A-S\|$ is either unique or has infinitely many values. Thus, we have:

Corollary 4. Let $A$ be a partial Toeplitz contraction whose strict upper triangular part is known, and assume that one of its rectangular submatrices has norm 1 (called the extremal case). Let $\widetilde{A}$ be a Toeplitz contraction of $A$ such that $\widetilde{A}-A$ is a contraction itself. Then $t c_{A}$ is either a singleton or an infinite set.

Example 5. Let $T=\left(\begin{array}{ll}b & a \\ x & b\end{array}\right) \in M_{2}(\mathbb{C})$. By the preceding considerations there exists $x \in \mathbb{C}$ such that min $\|T\|=\left\|\left(\begin{array}{l}a \\ b\end{array}\right)\right\|=\sqrt{|a|^{2}+|b|^{2}}$. Corollary 4 shows that $x$ is unique or there are infinitely many solutions $x$. Indeed, if $b \neq 0$, then $x=-\frac{b^{2}}{|b|^{2}} \bar{a}$ is the only solution that minimizes $\|T\|$, and if $b=0$, then all values $x$ in the disk $|x| \leq|a|$ minimize $\|T\|$.

Remark 6. (i) We need not expect that the collection $\mathfrak{G}_{N}$ of $N \times N$ Toeplitz contractions is strictly convex with respect to the collection of $N \times N$ Toeplitz matrices; for example, take $C:=\left(\begin{array}{ll}1 & 0 \\ 0 & 1\end{array}\right)$ and $D:=\left(\begin{array}{ll}0 & 1 \\ 1 & 0\end{array}\right)$, and observe that $C, D \in \partial \mathfrak{G}_{N}$ and also $\frac{1}{2}(C+D) \in \partial \mathfrak{G}_{N}$ because $\left\|\frac{1}{2}(C+D)\right\|=\left\|\left(\begin{array}{cc}\frac{1}{2} & \frac{1}{2} \\ \frac{1}{2} & \frac{1}{2}\end{array}\right)\right\|=1$. Also, the collection $\mathfrak{C}_{N}$ of $N \times N$ contractions is not strictly convex. In fact it is well known that ext $\mathfrak{C}_{N}$ is the set of unitaries. More generally, it is known (cf. [Ha Solution 136]) that the extreme points of the unit ball in the space of bounded linear operators on a Hilbert space are the maximal partial isometries, that is, the isometries and coisometries.

(ii) By (i) above,

$$
\operatorname{ext} \mathfrak{T}_{N} \bigcap \operatorname{ext} \mathfrak{C}_{N}=\left\{e^{i \theta} I_{N}: \theta \in[0,2 \pi)\right\},
$$

which also follows at once from the observation that unitary triangular Toeplitz matrices are rotations of the identity.

(iii) The Frobenius norm $\|\cdot\|_{2}$ is defined on $M_{n}$ by

$$
\|A\|_{2}:=\left(\sum_{i, j=1}^{n}\left|a_{i j}\right|^{2}\right)^{\frac{1}{2}} .
$$


Then $[A, B]:=\operatorname{tr}\left(A B^{*}\right)$ defines an inner product on $M_{n}(\mathbb{C})$, and the Frobenius norm is induced from $[\cdot, \cdot \cdot$. Thus, the Frobenius norm satisfies the parallelogram identity

$$
\frac{1}{2}\left(\|C+D\|_{2}^{2}+\|C-D\|_{2}^{2}\right)=\|C\|_{2}^{2}+\|D\|_{2}^{2} \quad\left(C, D \in M_{n}(\mathbb{C})\right) .
$$

Therefore, if $\|C\|_{2}=\|D\|_{2}=\left\|\frac{1}{2}(C+D)\right\|_{2}=1$, then it follows that $C=D$. This shows that $\mathfrak{T}_{N}$ is also strictly convex in the Frobenius norm.

\section{COWEn SETS}

In this section we will show how Theorem 1 can be applied to the study of Cowen's sets for analytic polynomials. We first need some definitions and preliminary results. A bounded linear operator $A$ on a Hilbert space $\mathfrak{H}$ with inner product $(\cdot, \cdot)$ is said to be hyponormal if its selfcommutator $\left[A^{*}, A\right]=A^{*} A-A A^{*}$ induces a positive semidefinite quadratic form on $\mathfrak{H}$ via $\xi \mapsto\left(\left[A^{*}, A\right] \xi, \xi\right)$, for $\xi \in \mathfrak{H}$. When the Hilbert space under consideration is the Hardy space $H^{2}(\mathbb{T})$ of the unit circle $\mathbb{T}=\partial \mathbb{D}$ in the complex plane, a natural collection of operators to study is the class of Toeplitz operators. Given $\varphi \in L^{\infty}(\mathbb{T})$, the Toeplitz operator $T_{\varphi}$ with symbol $\varphi$ is defined by $T_{\varphi} f:=P(\varphi \cdot f)$, where $f \in H^{2}(\mathbb{T})$ and $P$ denotes the orthogonal projection that maps $L^{2}(\mathbb{T})$ onto $H^{2}(\mathbb{T})$. An elegant theorem of C. Cowen Cow2 characterizes the hyponormality of a Toeplitz operator $T_{\varphi}$ on $H^{2}(\mathbb{T})$ in terms of properties of the symbol $\varphi \in L^{\infty}(\mathbb{T})$. We shall use a variant of Cowen's Theorem [Cow2], first proposed by Nakazi and Takahashi [NT].

Cowen's Theorem. For $\varphi \in L^{\infty}(\mathbb{T})$, write

$$
\mathcal{E}(\varphi):=\left\{k \in H^{\infty}(\mathbb{T}):\|k\|_{\infty} \leq 1 \text { and } \varphi-k \bar{\varphi} \in H^{\infty}(\mathbb{T})\right\} .
$$

Then $T_{\varphi}$ is hyponormal if and only if $\mathcal{E}(\varphi)$ is nonempty.

Cowen's method is to recast the operator-theoretic problem of hyponormality for Toeplitz operators into the problem of finding a solution of a certain functional equation involving its symbol. Suppose $\varphi$ is a trigonometric polynomial of the form $\varphi(z)=\sum_{n=-N}^{N} a_{n} z^{n}$, where $a_{N} \neq 0$. If $k(z)=\sum_{j=0}^{\infty} c_{j} z^{j}$ is a function in $H^{\infty}(\mathbb{T})$, then $\varphi-k \bar{\varphi} \in H^{\infty}(\mathbb{T})$ if and only if the coefficients $c_{0}, \cdots, c_{N-1}$ are given by

$$
\left(\begin{array}{c}
\overline{c_{0}} \\
\overline{c_{1}} \\
\vdots \\
\overline{c_{N-1}}
\end{array}\right)=\left(\begin{array}{ccccc}
a_{1} & a_{2} & \ldots & a_{N-1} & a_{N} \\
a_{2} & a_{3} & \ldots & a_{N} & 0 \\
\vdots & \vdots & \ddots & \vdots & \vdots \\
a_{N} & 0 & \ldots & 0 & 0
\end{array}\right)^{-1}\left(\begin{array}{c}
\overline{a_{-1}} \\
\overline{a_{-2}} \\
\vdots \\
\overline{a_{-N}}
\end{array}\right)
$$

(cf. [Zhu] $)$. By Cowen's Theorem, if $c_{0}, \cdots, c_{N-1}$ are given by (3), then the hyponormality of $T_{\varphi}$ is equivalent to the existence of a function $k \in H^{\infty}(\mathbb{T})$ such that

$$
\left\{\begin{array}{l}
\widehat{k}(j)=c_{j}, \quad j=0, \cdots, N-1, \\
\|k\|_{\infty} \leq 1,
\end{array}\right.
$$

which is precisely the formulation of CSIP. 
From the preceding considerations the following result follows at once.

Lemma 7. Suppose that $\varphi(z)=\sum_{n=-N}^{N} a_{n} z^{n}$, where $a_{N} \neq 0$ and let $c_{0}, \cdots, c_{N-1}$ be given by (3). Then $T_{\varphi}$ is hyponormal if and only if the Toeplitz matrix

$$
C:=\left(\begin{array}{ccccc}
c_{0} & 0 & 0 & \ldots & 0 \\
c_{1} & c_{0} & \ddots & & \vdots \\
\vdots & \ddots & \ddots & \ddots & \vdots \\
c_{N-2} & & \ddots & \ddots & 0 \\
c_{N-1} & c_{N-2} & \ldots & c_{1} & c_{0}
\end{array}\right)
$$

is a contraction. Moreover, if $\|C\|=1$, then $\mathcal{E}(\varphi)$ has precisely one element $k$ which is a finite Blaschke product of degree at most $N$.

We now introduce the notion of Cowen set.

Definition 8 (cf. Cow1, [Cow2]). Let $H=\left\{h \in H^{\infty}: h(0)=0\right.$ and $\left.\|h\|_{2} \leq 1\right\}$. For $f \in H^{2}$, let $G_{f}$ denote the set of $g$ in $H^{2}$ such that for every $h$ in $H^{2}$,

$$
\sup _{h_{0} \in H}\left|\left\langle h h_{0}, f\right\rangle\right| \geq \sup _{h_{0} \in H}\left|\left\langle h h_{0}, g\right\rangle\right| .
$$

We shall say that $G_{f}$ is the Cowen set for $f$.

We now see how this definition is relevant to hyponormality of Toeplitz operators. First, we need some notation. For $\varphi \in L^{\infty}(\mathbb{T})$, the Hankel operator $H_{\varphi}: H^{2} \rightarrow H^{2}$ is defined by $H_{\varphi} f:=J(I-P)(\varphi f)$, where $J:\left(H^{2}\right)^{\perp} \rightarrow H^{2}$ is given by $J z^{-n}=z^{n-1}$ for $n \geq 1$. Suppose now that $p \in H^{\infty}$ and $h \in H^{2}$. Then we have

$$
\begin{aligned}
\sup _{h_{0} \in H}\left|\left\langle h h_{0}, p\right\rangle\right|=\sup _{h_{0} \in H}\left|\left\langle\bar{p} h, \bar{h}_{0}\right\rangle\right| & =\sup _{h_{0} \in H}\left|\left\langle(I-P) \bar{p} h, \bar{h}_{0}\right\rangle\right| \\
& =\sup _{\substack{g \in H^{\infty} \\
\|g\|_{2} \leq 1}}\left|\left\langle(I-P) \bar{p} h, J^{*} g\right\rangle\right| \\
& =\sup _{\substack{g \in H^{\infty} \\
\|g\|_{2} \leq 1}}|\langle J(I-P) \bar{p} h, g\rangle|=\left\|H_{\bar{p}} h\right\| .
\end{aligned}
$$

Recall ([Cow1, Proposition 11]) that if $f, g \in H^{\infty}$ and $\varphi=f+\bar{g}$, then the following are equivalent:

(1) $T_{\varphi}$ is hyponormal;

(2) $\left\|H_{\bar{f}} h\right\| \geq\left\|H_{\bar{g}} h\right\|$ for every $h \in H^{2}$.

From Definition 8 and the above considerations we can see that if $f \in H^{\infty}$, then

$$
G_{f}=\left\{g \in H^{\infty}: T_{f+\bar{g}} \text { is hyponormal }\right\} .
$$

In Cow2], it was shown that $G_{f}$ is balanced, convex, and weakly compact. Also, recall $([\mathrm{FL}])$ that if $\varphi(z)=\sum_{n=-m}^{N} a_{n} z^{n}$ is such that $T_{\varphi}$ is hyponormal, then $m \leq N$ and $\left|a_{-m}\right| \leq\left|a_{N}\right|$. Thus, if $f$ is an analytic polynomial of degree $N$, then

$G_{f}=\{g: g$ is an analytic polynomial of degree at most $N$ and $T_{f+\bar{g}}$ is hyponormal\}. 
Since the hyponormality of $T_{\varphi}$ is independent of the Fourier coefficient $\hat{\varphi}(0)$, we assume, without loss of generality, that $g(0)=0$. Therefore, if $f(z):=\sum_{n=0}^{N} a_{n} z^{N}$, it is more convenient to work with the set

$$
G_{f}^{\prime}=\left\{g(z):=b_{1} z+\cdots+b_{N} z^{N}: T_{f+\bar{g}} \text { is hyponormal }\right\} .
$$

We shall call $G_{f}^{\prime}$ the reduced Cowen set for $f$. We easily see that $G_{f}^{\prime}$ is convex and compact in the norm topology of $H^{\infty}$.

In 1988, Cowen posed the following:

Problem ([Cow1, Question 3]). What are the extreme points of $G_{f}^{\prime}$ ? In particular, if $g \in G_{f}^{\prime}$ but $\lambda g \notin G_{f}^{\prime}$ for all $|\lambda|>1$, is $g$ an extreme point of $G_{f}^{\prime}$ ?

We now answer the above problem for the case of analytic polynomials.

Theorem 9. If $f$ is an analytic polynomial of degree $N$, then $G_{f}^{\prime}$ is strictly convex and

$$
\operatorname{ext} G_{f}^{\prime}=\left\{g(z)=b_{1} z+\cdots+b_{N} z^{N}:\|C\|=1\right\},
$$

where $C$ is the Toeplitz matrix in (5) corresponding to $\varphi=f+\bar{g}$.

Proof. Suppose $\varphi \equiv f+\bar{g}$ with $f(z) \equiv \sum_{j=0}^{N} a_{j} z^{j}$ and $g(z) \equiv \sum_{j=1}^{N} b_{j} z^{j}$, and write $G_{f}^{\prime}:=\left\{g: T_{\varphi}\right.$ is hyponormal $\}$. By the preceding considerations, $G_{f}^{\prime}$ is convex and compact. We must show that $\partial G_{f}^{\prime} \subseteq \operatorname{ext} G_{f}^{\prime}$. Suppose that $k(z)=\sum_{j=0}^{\infty} c_{j} z^{j}$ is in the closed unit ball of $H^{\infty}(\mathbb{T})$, where $c_{0}, \cdots, c_{N-1}$ are given by (3). Write

$$
\mathfrak{h}_{N}:=\left\{\left(c_{0}, \cdots, c_{N-1}\right) \in \mathbb{C}^{N}: T_{\varphi} \text { is hyponormal }\right\} .
$$

If $F: b_{1} z+\cdots+b_{N} z^{N} \mapsto\left(b_{1}, \cdots, b_{N}\right)$ is a canonical map from the set of polynomials $g$ of degree at most $N$ with $g(0)=0$ onto $\mathbb{C}^{N}$ and if $L$ is the inverse of the Hankel matrix in (3), then $L F\left(G_{f}^{\prime}\right)=\overline{\mathfrak{h}_{N}}$, where $\overline{\mathfrak{h}_{N}}$ denotes the set of conjugates of elements in $\mathfrak{h}_{N}$. Note that $L F$ is bijective. By Con Proposition V.7.9], every bijective bounded linear operator between Hilbert spaces preserves compactness, convexity, the topological boundary, and the set of extreme points, so it suffices to show that $\partial \mathfrak{h}_{N} \subseteq \operatorname{ext} \mathfrak{h}_{N}$. By Lemma 7 , we know that $\mathfrak{h}_{N}=\left\{\left(c_{0}, \cdots, c_{N-1}\right) \in\right.$ $\left.\mathbb{C}^{N}:\|C\| \leq 1\right\}$, so that the result follows at once from Theorem 1.

\section{ACKNOWLEDGMENTS}

We are thankful to Professor Carl Cowen for helpful comments on the topics in this article. We are also grateful to the referee, for several suggestions that improved the presentation.

\section{REFERENCES}

[Con] J.B. Conway, A Course in Functional Analysis, Springer-Verlag, New York, 1985. MR 86h:46001

[Cow1] C.C. Cowen, Hyponormal and subnormal Toeplitz operators, Surveys of Some Recent Results in Operator Theory, I (J.B. Conway and B.B. Morrel, eds.), Pitman Research Notes in Mathematics, Vol 171, Longman, 1988, pp. 155-167. MR 90j:47022

[Cow2] C.C. Cowen, Hyponormality of Toeplitz operators, Proc. Amer. Math. Soc. 103 (1988), 809-812. MR 89f:47038

[Da] K.R. Davidson, Nest Algebras, Pitman Res. Notes Math. Ser., Vol 191, Longman, 1988. MR 90f:47062

[dLR] K. deLeeuw and W. Rudin, Extreme points and extremal problems in $H_{1}$, Pacific J. Math. 8 (1958), 467-485. MR 20:5426 
[FL] D.R. Farenick and W.Y. Lee, Hyponormality and spectra of Toeplitz operators, Trans. Amer. Math. Soc. 348 (1996), 4153-4174. MR 97k:47027

[Ga] J.B. Garnett, Bounded Analytic Functions, Academic Press, New York, 1981. MR 83g:30037

[GGK] I. Gohberg, S. Goldberg and M.A. Kaashoek, Classes of Linear Operators, Vol II, Birkhäuser Verlag, Basel, 1993. MR 95a:47001

[JR] C.R. Johnson and L. Rodman, Completion of Toeplitz partial contractions, SIAM J. Matrix Anal. Appl. 9 (1988), 159-167. MR 89f:47040

[Ha] P.R. Halmos, A Hilbert Space Problem Book, Springer-Verlag, New York, 1982. MR 84e:47001

[NT] T. Nakazi and K. Takahashi, Hyponormal Toeplitz operators and extremal problems of Hardy spaces, Trans. Amer. Math. Soc. 338 (1993), 753-767. MR 93j:47040

[Næ] G. Nævdal, On the completion of partially given triangular Toeplitz matrices to contractions, SIAM J. Matrix Anal. Appl. 14 (1993), 545-552. MR 94a:47025

[Sch] I. Schur, Über Potenzreihen die im Innern des Einheitskreises beschränkt, J. Reine Angew. Math. 147 (1917), 205-232.

[Ta] S. Takahashi, Extension of the theorems of Carathéodory-Toeplitz-Schur and Pick, Pacific J. Math. 138 (1989), 391-399. MR 90d:30105

[Zhu] K. Zhu, Hyponormal Toeplitz operators with polynomial symbols, Integral Equations Operator Theory 21 (1995), 376-381. MR 95m:47044

Department of Mathematics, Kanagawa University, Yokohama 221-8686, Japan

E-mail address: chiyom01@kanagawa-u.ac.jp

Department of Mathematics, University of Iowa, Iowa City, Iowa 52242

E-mail address: curto@math.uiowa.edu

Department of Mathematics, SungKyunKwan University, Suwon 440-746, Korea

E-mail address: wylee@yurim.skku.ac.kr 